\section{S40 TNF $\alpha$ REDUCES BMPR-II EXPRESSION BUT ENHANCES BMP6 SIGNALLING VIA ACTR-IIA IN PULIMONARY ARTERIAL SMOOTH MUSCLE CELLS}

doi:10.1136/thoraxjnl-2012-202678.046

LA Hurst, AA Rana, NW Morrell, PD Upton. Department of Medicine, University of Cambridge, Cambridge, United Kingdom

Mutations in the bone morphogenetic protein type-II receptor (BMPR-II) underlie $~ 70 \%$ of heritable pulmonary arterial hypertension (PAH) cases. However, the low penetrance in mutation carriers implies a 'second hit' is required for disease onset. A possible trigger is inflammation. Circulating tumour necrosis factor alpha (TNF $\alpha)$ is raised in PAH patients and TNF $\alpha$ can alter smooth muscle cell proliferation. Thus, we examined the effect of TNF $\alpha$ on BMP signalling.

Human pulmonary arterial smooth muscle cells (PASMCs) were derived from patients with BMPR-II mutations and from diseasefree controls. Quantitative polymerase chain reaction (qPCR) and western blotting revealed that 24 hours of TNF $\alpha$ stimulation reduced the expression of BMP2 and BMPR-II but increased BMP6 expression. TNFa reduced BMP2-mediated signalling, as expected. In contrast, BMP6 treatment in the presence of TNF $\alpha$ increased smad1/5 activation and upregulated ID1 expression, implying that BMP6 can signal independently of BMPR-II Furthermore, BMPR-II mutant PASMCs proliferated in response to BMP6 and TNF $\alpha$. Quantitative PCR revealed increased ActR-IIa expression following TNF $\alpha$ stimulation and siRNA knockdown of ActR-IIa abrogated this enhanced proliferative response.

In summary, TNF $\alpha$ increases BMP6 expression, which in the absence of functional BMPR-II, can bind to receptor complexes involving ActR-IIa to accelerate PASMC proliferation. The potential contribution of this novel mechanism to vascular remodelling in the systemic and pulmonary circulation requires further study.

\section{S41 CONDUCTANCE-DERIVED RIGHT VENTRICULAR STROKE WORK MEASURED BY PRESSURE VOLUME LOOPS IN CHRONIC THROMBOEMBOLIC PULMONARY VASCULAR DISEASE}

doi:10.1136/thoraxjnl-2012-202678.047

C McCabe, D Taboada, R Mackenzie-Ross, I Harvey, K Sheares, P White, R Axell, S Hoole, L Shapiro, J Pepke-Zaba. Papworth Hospital, Cambridge, United Kingdom

Background The most significant determinant of both symptoms and survival in pulmonary arterial hypertension (PAH) is the degree of cardiac impairment that results from increased right ventricular after load.[1] Resting haemodynamic parameters however do not always correlate with exercise symptoms and performance limitation. Pulmonary vascular resistance (PVR) is normally measured at rest and is derived from mean flow data. This fails to take into account both the pulsatile nature of flow in the pulmonary artery and thus pulmonary arterial compliance. [2]

Chronic thromboembolic pulmonary hypertension (CTEPH) lies within Group IV of the Dana Point 2008 Classification and arises from persistent pulmonary vascular occlusion secondary to arterialthrombus. However, for unclear reasons a proportion of patients with significant thrombus burden do not develop elevation of pulmonary arterypressures creating a two compartment model of disease. Precise haemodynamic abnormalities are likely to be determined by several factors including thrombuschronicity, load, distribution, pulmonary artery compliance and right ventricular adaptation in response to increased after load though there is no discernible relationship between routinely measured haemodynamics and any of these parameters. Furthermore, the effect of right ventricular performance on exercise capacity remains poorly characterised in this disease subgroup.

Aims and Methods We hypothesise that exercise capacity in ourpatient cohort ultimately depends on the adaptation of both right ventricle and pulmonary arteries to thrombotic insult. Using a conductance-based pressure volume approach at the time of right heart catheterisation, we measured the right ventricular mechanoenergetics in six patients ( 2 female) with chronic pulmonary vascular occlusion but different resting haemodynamics. These results were compared with peak exercise oxygen consumption assessed by contemporaneous cardiopulmonary exercise testing.

Results and Conclusions Peak exercise capacity (peak $\mathrm{VO}_{2}$ )was inversely related to PVR throughout our patient group $(p=0.046)$. The close association between PVR and right ventricular stroke work index (RVSWI) $(\mathrm{r}=0.96)$, derived from the area within the pressure volume loop (Fig 1), suggests an increased resting stroke work may result in earlier cardiovascular limitation at peak exercise in those with more severe pulmonary hypertension. This seems physiologically compatible given the greater after load seen with more severe pulmonary hypertension and diminished cardiac reserve. Initial analysis of isovolumic relaxation indices $\left(\mathrm{Tau}, \mathrm{dP} / \mathrm{dt}_{\min }\right.$ ) suggest pulmonary hypertension is not mediated by diastolic dysfunction within the right ventricle and may be more related to reduced pulmonary artery compliance(Ea). The impaired right ventricular stroke work for low/normal PVR fits with patient symptoms and provides insight into the early stages of the process of right ventricular failure.

1. Girgis RE Predicting long term survival in pulmonary arterial hypertension JACC 20115824 p2520.

2. Chesler et al. How to measure pulmonary vascular and right ventricular function. Conf Proc IEEE Eng Med Biol Soc 2009 p177.

\section{Sleep apnoea diagnosis and consequence}

\section{S42 TWO YEAR VASCULAR EVENT, HOSPITAL ADMISSIONS, AND ACCIDENT INCIDENCE: FURTHER DATA FROM THE MOSAIC SLEEP APNOEA TRIAL}

doi:10.1136/thoraxjnl-2012-202678.048

${ }^{1}$ CD Turnbull, ${ }^{1 S}$ Craig, ${ }^{2 M}$ Kohler, ${ }^{1 D}$ Nicoll, ${ }^{1} \mathrm{~J}$ Stradling. 'Oxford Sleep Unit, Churchill Hospital, Oxford, United Kingdom; ${ }^{2}$ Sleep Disorders Centre and Pulmonary Division, University Hospital, Zurich, Switzerland

Introduction. The MOSAIC multicentre trial investigated the effect of CPAP versus no CPAP on both sleepiness and a vascular risk score, over 6 months, in nearly 400 minimally symptomatic patients with OSA. The results on the vascular risk score, reported previously, suggested no potential vascular benefit of CPAP, although endothelial function improved. The incidence of real events is needed to calculate the size of any future trials in this group of patients.

\begin{tabular}{|c|c|c|c|c|c|c|c|c|c|c|c|c|c|}
\hline Sex & Age & $\begin{array}{c}\text { NYHA } \\
\text { Class }\end{array}$ & $\begin{array}{l}\text { mPAP } \\
\text { mmHg }\end{array}$ & $\underset{2}{\text { L.m }}$ & $\begin{array}{c}\text { PVR } \\
\text { Dyn }\end{array}$ & $\underset{\text { minn }^{-1}}{\text { HR }}$ & $\begin{array}{l}\text { Tau } \\
\text { ms }\end{array}$ & $\underset{\text { mmHg }}{\mathbf{P}_{\text {max }}}$ & $\begin{array}{c}\text { RVSwI } \\
\text { mmHgeml.m }\end{array}$ & 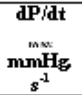 & $\begin{array}{c}\mathrm{dP} / \mathrm{dt} \\
\mathrm{min} \\
\mathrm{mmH} \\
s^{-1}\end{array}$ & $\begin{array}{c}\mathbf{E a} \\
\underset{\mathbf{L}^{-1}}{\min \mathbf{m}} \mathbf{m}\end{array}$ & $\begin{array}{c}\text { peakv02 } \\
\text { ml min } \\
{ }^{1} \cdot \mathrm{Kg}^{-1}\end{array}$ \\
\hline $\mathrm{M}$ & 28 & II & 32 & 2.2 & 417 & 61.2 & 38.5 & 906 & 2401 & 912 & -972 & 1.34 & 20.2 \\
\hline $\mathrm{F}$ & 45 & II & 55 & 2.5 & 700 & 81.3 & 191 & 842 & 4730 & 1789 & -860 & 1.37 & 18.0 \\
\hline$M$ & 60 & II & 35 & 3.1 & 288 & 54.5 & 94.6 & 58.4 & 733 & 625 & -646 & 0.96 & 22.8 \\
\hline$M$ & 49 & II & 33 & 2.6 & 301 & 51.2 & 71.4 & 603 & 1779 & 408 & -592 & 0.69 & 22.1 \\
\hline$M$ & 61 & II & 15 & 2.3 & 157 & 61.1 & 65.3 & 435 & 591 & 601 & -522 & 0.63 & 29.7 \\
\hline $\mathrm{F}$ & 46 & II & 44 & 2.5 & 661 & 85.0 & 42.0 & 80.1 & 3564 & 510 & -205 & 1.47 & 19.2 \\
\hline
\end{tabular}

Abstract S41 Figure 1 